Journal of Neurology Research Reviews \& Reports

\title{
Clinical Study of Chronic Heredofamilial Migraine with
} Aura

\author{
Orlando J. Castejón ${ }^{1^{*}}$, Galindez, $\mathrm{P}^{2}$, De Castejón Salones $\mathrm{M}^{1,3}$, Torres IA ${ }^{2}$ \\ ${ }^{1}$ Faculty of Medicine, Zulia University \\ ${ }^{2}$ Biological Research Institute \\ ${ }^{3}$ Faculty of Medicine, Zulia University
}

\begin{abstract}
Sixteen patients ranging from 19 to 68 years-old were studied with Chronic Heredofamilial Migraine with Aura. The following symptoms were associated to chronic heredofamilial migraine: dizziness, vertigo, syncope, redness eye, photophobia, blurred vision and reduced and loss of vision, scintillant scotomas, dizziness, ears noise, temporospatial disorientation, memory disorders, effort dyspnea, asthenia, son phobia, nausea, vomits, and social, labor and home stress. The followings symptoms were interpreted as migraine auras: Blurred vision and reduced and loss of vision, scintillant scotomas, dizziness, ears noise. The following comorbidities were found: syncope, bradycardia, mitral prolapse and tachycardia, effort dyspnea, asthenia, seizures, environment, labor and home stress, sleep disorders or insomnia and constipation. The followings aspects were discussed: migraine and prodroms, migraine and vascular diseases, migrainous vertigo and dizziness, symptoms related to the visual system in migraine, migraine and sleep disorders, hemiplegic migraine, neural correlates of migraine, histamine and migraine, the calcitonin gene-related peptide in migraine and its role in migraine physiopathology, and migraine genetic.
\end{abstract}

Corresponding author: Orlando J.Castejón, Instituto de Investigaciones Biológicas. Apdo. 526. Maracaibo. Venezuela. Cell Phone 58-424-613-8009, Email: ocastejo@cantv.net

Received: December 19, 2019, Accepted: January 15, 2020, Published: January 20, 2020

Keywords: Heredofamilial Migraine, Migraine Comorbidities, Migraine Genetic

\section{Introduction}

Migraine is a complex neurovascular disorder with a strong genetic component. However, convincingly demonstrated genetic findings in other primary headaches such as cluster headache and tension-type headache are even rarer [1]. Migraine is clinically divided into two main subtypes that are based on the absence or presence of an aura: migraine without aura (MO) and migraine with aura (MA) [2]. Headache associated with cerebrovascular diseases lacks clear-cut characteristics as it may resemble a migraine attack, tension-type headache, or cluster headache [3]. The vast majority of headache patients encountered in the outpatient general medicine setting will be diagnosed with a primary 
headache disorder, mostly migraine or tensiontype headache. Nonmigraine primary headaches include trigeminal autonomic cephalalgias, primarily cluster headache; facial pain, primarily trigeminal neuralgia; and miscellaneous headache syndromes, such as hemicrania continua and new daily persistent headache [4]. According to migraine is a common neurovascular disease associated with vascular risks, especially in young adult females, but the mechanism underlying these associations remains unknown [5]. A few factors that influence migraine or comorbidities are sleep, obstructive sleep apnea (OSA), obesity, and affective disorders, as well as autoimmune diseases [6]. In the present study we study from the clinical point of view, in a developing country, 16 patients with chronic heredofamilial migraine with aura and the underpinning physiopathological mechanisms.

\section{Material and Methods}

Sixteen patients ranging from 19 to 68 years-old were studied at the Clinical Neuroscience Outpatient Clinic of Clinical Neuroscience Institute. Maracaibo. VenezuelaSan Rafael Clinical Home and the Biological Research Institute. Faculty of Medicine, Zulia University. Chronic heredofamilal migraine was diagnosed according to the International Headache Society-IIIb criteria.

\section{Results}

Case Report Summaries Case 1. FP, 29 years-old, F. Chronic migraine, sleep disorders, numbness or paresthesias of legs, history of family headaches, social stress by an attack from social delinquents. Family history of migraine.

Diag: Heredofamilal migraine. Stress Case 2. EB, 48 years-old, F. Chronic headache since 20 years ago, nausea, redness eye, memory disorders, temporospatial disorientation. Normal blood pressure, Bradycardia. Polycystic ovary syndrome.

Diag: Chronic migraine, Bradycardia. Polycystic ovary syndrome.

Case 3. MA, 27 years old, F. Fronto-parietal headache since 15 years old, referred to neck, seizures, labor stress, sleep disorders. Family history of migraine

Diag: Chronic heredofamiliar migraine. Stress Case 4. YS, 35 years old, F. Occipital headache, dizziness, vertigo, blurred vision, scintillant scotomas, Family history of migraine. Exertional dyspnoea. Family stress, normal blood pressure, Mitral prolapse and tachycardia, Family history of migraine

Diag: Chronic heredofamiliar migraine, Exertional dyspnoea, Mitral prolapse and tachycardia. Case 5. CI, 46 years old, F. Chronic migraine since 10 years ago, and syncope, photophobia, sonophobia, insomnia, Family history of migraine.

Diag: Chronic heredofamilial migraine, insomnia. Case 6. AY, 41 years old. F. Chronic frontal headache referred to temporal region and neck since 30 years ago, syncope, vertigo, loss of vision on right eye. Family history of migraine. Family history of migraine.

Diag: Chronic heredofamilial migraine, syncope, vertigo. Case 7, AMS.36 years old, F. Migraine since 20 years ago, blurred vision, scintillant scotomas, photophobia, and nausea. Family history of migraine.

Diag: Chronic heredofamilial migraine, vision disorder, nausea. Case 8, BP, 68 years old, F. Chronic migraine since 18 years ago, headache referred to neck and shoulders, redness eye, insomnia. Cerebrovascular accident 11 years ago, left hemiparesis, constipation, and Family history of migraine.

Diag: Chronic heredofamilial migraine, insomnia, constipation. Previous cerebrovascular accident. Family history of migraine. Case 9. MAV, 30 years old, F. Chronic migraine since 15 years ago, syncope and ears noise. The headache is initiated at the retroauricular region and ascent to parietal region. Family history of migraine.

Diag: Chronic heredofamilial migraine, syncope. Case 10. KM, 38 years old, F. Chronic Migraine. Cervical headache referred to arms and shoulders, dizziness, reduced visual acuity, nausea, asthenia, backward muscle contraction, sleep disorders, Family history of migraine.

Diag: Chronic heredofamilial migraine, dizziness, reduced visual acuity, nausea. Case 11 . HN, 19 years old, F. Chronic migraine since 7 years ago, redness eyes, scintillant scotomas, vomits, dizziness, vertigo. Family history of migraine. Family history of migraine.

Diag. Chronic heredofamiliar migraine, dizziness, insomnia.Case 13. AP, 37 years old, F, chronic headache at right hemicraneal region since 18 years ago, mainly at evening and night, redness eyes and stress at home. 
Family history of migraine.

Diag: Chronic heredofamiliar migraine, stress. Case 14. AL, 56 years old, F. Chronic headache at right hemicraneeal region since two years ago, headache, vomits, dizziness, depression and tremor. Family history of migraine.

Diag. Chronic heredofamilair migraine, dizziness, tremor, depression. Case 15. AA, 26 years old, F. Headache, redness eyes, sleep disorder, anxiety, hyperthyroidism, and labor stress. Family history of migraine.

Diag. Chronic migraine, hyperthyroidism, anxiety. Case 16. JG, 32 years old, M, Holocraneal headache since one week ago, dizziness. Family history of migraine.

\section{Interpretation of Results}

We have found the following symptoms associated to chronic heredofamilial migraine: dizziness, vertigo, syncope, redness eye, photophobia, blurred vision and reduced and loss of vision, scintillant scotomas, dizziness, ears noise, temporospatial disorientation, memory disorders, scintillant scotomas, effort dyspnea, asthenia, sonophobia, nausea, vomits, and social, labor and home stress.

The followings symptoms were interpreted as migraine auras: Blurred vision and reduced and loss of vision, scintillant scotomas, dizziness, ears noise. The following comorbidities were found: syncope, bradycardia, mitral prolapse and tachycardia, effort dyspnea, asthenia, seizures, hyperthyroidism, polycystic ovary syndrome, environmental, labor and home stress, sleep disorders or insomnia and constipation.

\section{Discussion}

\section{Migraine and Prodroms}

Understanding the premonitory (prodromal) phase of migraine during which patients experience symptoms, such as yawning, tiredness, cognitive dysfunction, and food cravings may help explain apparent migraine triggers in some patients, thus offering better selfmanagement [7].

\section{Migraine and Vascular Diseases}

We have above reported four cases with associated vascular diseases. Epidemiological studies have shown a clear correlation between migraine and vascular disease in more and more patients. Pathophysiological studies show the relevance of the hypothalamus in the generation of migraine attacks. Glutamate seems to play an important role [8].

Familial hemiplegic migraine (FHM) falls within the category of migraine with aura. In migraine with aura (including familial hemiplegic migraine) the neurologic symptoms of aura are unequivocally localizable to the cerebral cortex or brain stem and include visual disturbance (most common), sensory loss (e.g., numbness or paresthesias of the face or an extremity), dysphasia and difficulty with speech. Approximately 40\%-50\% of families with FHM1 have cerebellar signs ranging from nystagmus to progressive, usually lateonset mild ataxia. Cerebral infarction and death have rarely been associated with hemiplegic migraine. Three genes are known to be associated with FHM: CACNA1A (FHM1), ATP1A2 (FHM2), and SCN1A (FHM3) [9].

\section{Migrainous Vertigo and Dizziness}

In the present study we have reported four cases with vertigo and dizziness. Migraine-associated vertigo is a vestibular disorder which manifests itself with spontaneous or positional rotational vertigo or dizziness induced by head motion. The vertigo may occur without accompanying headache and may last from seconds to several weeks. Migraine-associated vertigo can be diagnosed according to the following criteria: 1. Recurrent vestibular symptoms, 2. Migraine according to the criteria of the International Headache Society, 3. Migrainous symptoms during the vertigo, such as headache, photophobia, phonophobia, scintillating scotoma or other auras, 4. Exclusion of other causes. The mechanism of migraine-associated vertigo is still obscure. Several hypotheses relating to the pathophysiology of migraine have been proposed: cortical spreading depression, regional changes in brain perfusion, release of neurotransmitters and paroxysmal dysfunction of ion channels. Clinical findings suggest both central and peripheral vestibular involvement [10].

Migrainous vertigo (MV) is a vestibular syndrome caused by migraine and presents with attacks of spontaneous or positional vertigo lasting seconds to days and migrainous symptoms during the attack. 
MV is the most common cause of spontaneous recurrent vertigo and is presently not included in the International Headache Society classification of migraine. Dizziness may also be due to orthostatic hypotension, anxiety disorders or major depression which all have an increased prevalence in patients with migraine [11].

A strong association exists between vertigo and migraine, with MV being the most common cause of spontaneous (nonpositional) episodic vertigo. Symptoms can be quite variable among patients and within individual patients over time, creating a diagnostic challenge. MV generally presents with attacks of spontaneous or positional vertigo lasting seconds to days with associated migrainous symptoms. Better elucidation of the neurologic linkages between the central vestibular pathways and migraine-related pathways and the discovery of ion channel defects underlying some causes of familial migraine, ataxia, and vertigo have furthered the understanding of $\mathrm{MV}$ pathophysiology [12].

\section{Symptoms Related to the Visual System in Migraine}

In the present study we found seven patients with visual system alterations. Patients with "visual snow" suffer from continuous TV-static-like tiny flickering dots in the entire visual field. Most patients describe a syndrome with additional visual symptoms of the following categories: palinopsia ("afterimages" and "trailing"), entopic phenomena arising from the optic apparatus itself (floaters, blue field entoptic phenomenon, photopsia, self-light of the eye), photophobia, nyctalopia (impaired night vision), as well as the non-visual symptom tinnitus. The high prevalence of migraine and typical migraine aura in this population has led to the assumption that "visual snow" is caused by persistent migraine aura. The findings support the view that "visual snow," migraine, and typical migraine aura are distinct syndromes with shared pathophysiological mechanisms that need to be addressed in order to develop rational treatment strategies for this disabling condition [13].

Migraine shows disrupts vision more than any other motor or sensory function. The possible visual aura symptoms vary from occasional small flashes of light to complex visual hallucinations, the stereotyped teichopsia being the most typical pattern. It is unclear as to why aura occurs serendipitously, sometimes preceding, but also occurring after the headache, and why aura can present with multiple phenotypes. Migraine is a common headache disorder characterized by often-severe headaches that may be preceded or accompanied by a variety of visual symptoms. Although a typical migraine aura is not difficult to diagnose, patients with migraine may report several other visual symptoms, such as prolonged or otherwise atypical auras, "visual blurring", "retinal migraine", "ophthalmoplegic migraine", photophobia, palinopsia, and "visual snow" [14-15].

The visual system is involved in different ways in migraine. Visual auras are the most common form of migraine aura. It may consist of positive or negative visual symptoms and cortical spreading depression is felt to be the phenomenon that underlies it. Even in migraine without aura, vision it is not totally excluded given that one of the major criteria for the diagnosis of migraine is photophobia. In persistent visual aura, patients refer symptoms defined as visual snow and television static. In retinal migraine unilateral decreased vision or complete visual loss occurs. Ophthalmoplegic migraine is characterized by palsy of one among the three ocular motor nerves. Migraine visual aura, particularly when occurring without headache, is a diagnosis of exclusion [16].

\section{Migraine and Sleep Disorders}

We have found three patients with sleep disorders. The relationship between headache and sleep is complex and runs in two directions. Headache may be the consequence of a (primary or secondary) sleep disorder or its cause (in chronic tension-type headache and/or chronic migraine with or without painkiller abuse). It can also be related to sleep in an intrinsic way, as in the case of hypnic headache (which only appears during sleep) or other primary headaches, such as migraine and certain trigeminal-autonomic cephalgias (which can appear during the waking state or during sleep); this type of headache occurs mostly during REM sleep. The neural pathways that control sleep and pain are anatomically, physiologically and neurochemically 
cross-linked. These neural systems are located in the brain stem, the hypothalamus and the basal brain. Such cross-links are produced on two different levels. On the one hand, they occur in the serotoninergic nuclei of the brain stem, whose activity in physiologically diminished during REM sleep and which are involved in antinociceptive control. On the other hand, they are also to be found in the hypothalamus, where serotoninergic terminals have been detected in the suprachiasmatic nucleus (SCN). As cells in the SCN are lost with age, their circadian and homeostatic functioning fails, the activity of the hypothalamus-pineal axis is reduced and the endogenous melatonin rhythm is altered [17].

Circadian rhythm has important implications in primary headache disorders. Migraine attacks are known to be triggered by either excess or lack of sleep, from the anatomical perspective, there are close fiber connections between the hypothalamus and the trigeminal nucleus caudalis, thus reinforcing the importance of diurnal rhythm disturbance in the pathophysiology of headache disorders [18].

\section{Hemiplegic Migraine}

In our study we found just one case of migraine with hemiparesis. Hemiplegic migraine is a rare form of migraine in which the migraine headache attack is accompanied by unilateral weakness. Typically, migraine aura has visual symptoms, but motor symptoms are rare. Hemiplegic migraine is a rare subtype of migraine with aura, characterized by the presence of motor weakness as aura manifestation. The motor weakness is often accompanied by other forms of aura like impairment in vision, speech, or sensation. A hemiplegic migraine can run in the family (familial hemiplegic migraine) or can occursporadicallyin oneindividual (sporadichemiplegic migraine). Familial Hemiplegic Migraine (FHM) is a rare, autosomal-dominant subtype of hemiplegic migraine that runs in the family. The diagnostic criteria for familial hemiplegic migraine require that at least one first or second-degree relative has had attacks fulfilling the diagnostic criteria for hemiplegic migraine. International Classification of Headache Disorders-3 classifies FHM into 4 subtypes based on the genetic mutation associated with them. FHM1 is associated with mutations in the CACNA1Agene. FHM1 is the most common type and accounts for around $50 \%$ of cases of FHM. FHM1 is commonly associated with cerebellar degeneration. Mutations in the ATP1A2 gene cause FHM2, and it accounts for less than $25 \%$ of cases of FHM. Mutations in the SCN1A gene cause FHM 3. FHM4 is diagnosed if no known genetic mutation linked to FHM is identified. Sporadic Hemiplegic Migraine occurs only in one individual without a family connection. The characteristic feature of hemiplegic migraine is the presence of unilateral motor weakness as aura manifestation in at least some attacks. Motor weakness is however not the only type of aura present during the hemiplegic migraine attack. Other typical aura symptoms like visual field defects, scotoma, hemianopia, tingling, numbness, ataxia, fever, or lethargy can occur. Motor symptoms often start in the hand and gradually spread up into the arm and face. The unilateral weakness may switch sides between or during attacks. Rarely, patients may have a bilateral motor weakness either simultaneously or in succession. The symptoms usually occur over 20 to 30 minutes, although rarely, aura symptoms and motor weakness can develop acutely and can mimic a stroke [19].

\section{Neural Correlates of Migraine}

A putative role of the retino-hypothalamic-pineal (RHP) axis in the pathophysiology of primary headaches is reviewed in terms of (1) retinal dysfunction, (2) hypothalamic dysfunction and human circadian desynchrony, (3) pineal melatonin dysfunction and (4) rostral limbic dysfunction mediating the human stress response, The Author proposes The Unified RHP hypothesis, suggesting that an acute, periodic or chronic, circadian desynchrony and dysfunction of the whole or part of the RHP axis is implicated in the pathophysiology of primary headaches. Supportive evidence for the RHP hypothesis, including recent PET studies show changes in dorsal pons, hypothalamus and rostral limbic structures [20].

The hypothalamus forms part of the central autonomic network, regulating body homeostasis and controlling pain. To this effect, it is strongly wired to more rostral and caudal areas, in particular the brainstem periaqueductal grey, the locus coeruleus and the median raphe nuclei, all involved in autonomic and sleep mechanisms and also in the descending control of pain perception.

The hypothalamus, especially its posterior regions, becomes activated during attacks of the trigeminal autonomic cephalalgias (TACs), while brainstem, 
especially dorsal pontine, activity shows up during migraine attacks. The hypothalamus and interconnected brainstem areas likely represent the neural sites responsible for the chronobiological features of some headaches, in particular the sleep-related attacks typical of the TACs, migraines and the hypnic headaches [21]. The hypothalamus has emerged as a key brain area in several headache disorders including migraine and cluster headache. It is involved in homeostatic regulation, including pain processing and sleep regulation, enabling appropriate physiological responses to diverse stimuli. It is also a key integrator of circadian entrainment to light, in part regulated by pituitary adenylate cyclaseactivating peptide (PACAP). With its established role in experimental headache research, the peptide has been extensively studied in relation to headache in both humans and animals, however, there are only few studies investigating its effect on sleep in humans [22]. These endogenous systems, mainly originating from the brainstem, hypothalamus and cerebral cortex, are strongly influenced by behavioral, cognitive and emotional factors that are relevant for the survival of the individual. In disorders such as primary headaches, dysfunctions affecting brain regulation mechanisms contribute to the generation of episodic painful states in susceptible individuals, and to the evolution from acute to chronic migraine or cluster headache [23].

\section{Histamine and Migraine}

With its vasodilative effect, histamine was suggested early on to be involved in the pathophysiology of migraine and aggravate headache. The mechanisms for this are not known, and histamines do not penetrate the blood-brain barrier (BBB). However, circulating histamine may influence hypothalamic activity via the circumventricular organs that lack BBB. In the rat, prolonged activation of meningeal nociceptors induced by dural mast cell degranulation has been observed. Brain histamine is synthesized by neurons that are restricted to the posterior basal hypothalamus, more specific to the tuberomamillary nucleus (TMN), and that project practically to the whole central nervous system. The posterior hypothalamus is a suspected locus in quo in several primary headaches. Recently, a positron emission tomography study performed in the prodromal phase of migraine attacks supported the idea of initial involvement of this area. In another recent study, the thalamic nuclei receiving trigeminal output was also shown to have direct connections with the ventral TMN. The central histaminergic system plays an important role in the complex sleep-wake cycle, promoting cortical excitability during wakening and attention, and it consolidates the wake state. The period of the day, in the evenings and during the night, when there is reduced susceptibility for migraine attacks corresponds with less central histaminergic firing. Activation of both the $\mathrm{H} 3$ and the $\mathrm{H} 4$ receptor promotes inhibitory actions on neurons. The role of the central histaminergic system in migraine is largely unexplored, but findings from preclinical research may be linked to several aspects of the disorder. The histaminergic system of the brain may play an important role, especially in the initial phase of an attack, and histamine $\mathrm{H} 3$ and $\mathrm{H} 4$ receptor ligands may potentially have migraine prophylactic properties [23].

Histamine is an efficient inducer of migraine attacks in migraine patients by an $\mathrm{H} 1$ mechanism most likely extracerebrally. These findings merit further investigation of antihistamines in clinical drug trials, The H3 and $\mathrm{H} 4$ receptors are found in primarily in CNS and immune tissues, respectively. $\mathrm{H} 3$ is likely to be involved in antinociception and has been linked with cognitive, neurodegenerative and sleep disorders. With the current limited knowledge of the $\mathrm{H} 4$ receptor it is questionable whether or not the receptor is involved in migraine [24].

Histamine plays a crucial role in migraine pathogenesis sustaining the neurogenic inflammation pathway. Interaction between mast cells (MC) and calcitoningene related protein (CGRP) results in sensitization of trigeminal afferents and trigeminal ganglia (TG). Histamine binds with differing affinities to four different histaminergic G-protein coupled receptors, activating protein kinases, or triggering calcium release with subsequent mode of actions. The histamine system interacts with multiple regions in the CNS and may hypothetically modulate the migraine response. [25].

The neuropeptides substance $\mathrm{P}$, calcitonin gene-related peptide (CGRP) and vasoactive intestinal polypeptide (VIP) have been considered as important mediators 
in migraine and other primary headaches. CGRP and VIP have been found at increased concentrations in jugular venous plasma during attacks of migraine or cluster headache, and CGRP receptor antagonists have recently been shown to be effective in migraine therapy. Substance P and CGRP are produced from a subset of trigeminal afferents, whereas VIP derives from parasympathetic efferents. Release of these neuropeptides in the meninges can cause arterial vasodilatation, mast cell degranulation and plasma extravasation in animal experiments, but only CGRP seems to be relevant in migraine. Animal models have confirmed the important role of CGRP in meningeal nociception. The activity of spinal trigeminal neurons is a sensitive integrative measure of trigeminal activity and is partly under the control of CGRP, most likely via central mechanisms. CGRP released from central terminals of trigeminal afferents in the spinal trigeminal nucleus seems to facilitate nociceptive transmission via presynaptic mechanisms [26].

\section{The Calcitonin Gene-related Peptide in Migraine and its role in Migraine Physiopathology}

It has been observed in recent years that levels of such molecules as calcitonin gene-related peptide (CGRP) and, to a lesser extent, the pituitary adenylate cyclaseactivating peptide are elevated during migraine attacks and in chronic migraine, both in the cerebrospinal fluid and in the serum. The trigeminal ganglion plays a key role in primary headache pathophysiology. Calcitonin gene-related peptide (CGRP) and CGRP receptors are expressed in trigeminal neurons that form $\mathrm{C}$-fibers and A-fibers, respectively [27].

\section{Migraine Genetic}

As above stated migraine is a complex neurovascular disorder with a strong genetic component. Research into the genes involved in both types has provided insights into the many contributing genetic factors. Migraine is characterized into two main types, migraine without aura (MO) and migraine with aura (MA). Hemiplegic migraine is a rare monogenic MA subtype caused by mutations in three main genes - CACNA1A, ATP1A2 and SCN1A - which encode ion channel and transport proteins. Functional studies in cellular and animal models show that, in general, mutations result in impaired glutamatergic neurotransmission and cortical hyperexcitability, which make the brain more susceptible to cortical spreading depression, a phenomenon thought to coincide with aura symptoms. Variants in other genes encoding ion channels and solute carriers, or with roles in regulating neurotransmitters at neuronal synapses, or in vascular function, can also cause monogenic migraine, hemiplegic migraine and related disorders with overlapping symptoms [28].

Most progress has been made for Familial Hemiplegic Migraine, a rare subtype of migraine with aura. Three genes (CACNA1A, ATP1A2 and SCN1A) have been identified that all encode ion transporters [1-29].

Cellular and transgenic mouse studies suggest that neuronal hyperexcitability and increased susceptibility to cortical spreading depression, the correlate of migraine aura, are important molecular mechanisms in migraine. Investigating monogenic diseases in which migraine is a prominent feature such as CADASIL, which is caused by mutations in the NOTCH3 gene, can help understanding the pathology of migraine [1]. Mutations in these genes are also found in some patients with sporadic hemiplegic migraine. Linkage studies have identified several gene loci for the more common forms of migraine; however, identification of the respective causative genes is still pending $[29,30]$.

\section{Acknowledgment}

This paper has been carried out with a subvention from Castejon Foundation and the logistic support of Biological Research Institute. Faculty of Medicine. Zulia University and the Clinical Neuroscience Institute. Clinical Home San Rafael. Maracaibo Venezuela.

\section{References}

1. Van Den Maagdenberg AM, Terwindt GM, Haan J, Frants RR, Ferrari MD (2010) Genetics of headaches. Handb Clin Neurol 97: 85-97.

2. De Vries B, Frants RR, Ferrari MD, van den Maagdenberg AM (2009) Molecular genetics of migraine. Hum Genet126: 115-132.

3. Diamanti S, Longoni M, Agostoni EC (2019) Leading symptoms in cerebrovascular diseases: what about headache? 40:147-152. 
4. Vgontzas A, Rizzoli PB (2019) Nonmigraine headache and facial pain. Med Clin North Am 103: 235-250.

5. Yilmaz Avci A, Akkucuk MH, Torun E, Arikan S, Can U, al et (2019). Migraine and subclinical atherosclerosis: endothelial dysfunction biomarkers and carotid intima-media thickness: a case-control study Neurol Sci 40: 703-711.

6. Klenofsky B, Pace A, Natbony LR, Sheikh HU (2019) Episodic migraine comorbidities: avoiding pitfalls and taking therapeutic opportunities. Curr Pain Headache Rep 23:31.

7. Goadsby PJ, (2019) Primary headache disorders: Five new things. Neurol Clin Pract 9: 233-240.

8. Diener HC, Gaul C, Holle-Lee D, Jürgens TP, Kraya T, et al. (2019) Headache, an update. Laryngorhinootologie 98: 192-217.

9. Jen JC, Adam MP, Ardinger HH, Pagon RA, Wallace SE, et al. (2015) Gene Reviews Seattle (WA) University of Washington, Seattle 1993-2019.

10. Lempert T, Neuhauser H (2001). Vertigo as a symptom of migraine. Med Klin (Munich) 96: 475479.

11. Neuhauser H, Lempert T (2004) Vertigo and dizziness related to migraine: a diagnostic challenge 24 : 83-91.

12. Eggers SD (2006). Migraine-related vertigo: diagnosis and treatment. CurrNeurolNeurosciRep 6:106-115.

13. Schankin CJ, Maniyar FH, Sprenger T, Chou DE, Eller M, al et (2014) The relation between migraine, typical migraine aura and "visual snow" Headache 54: 957-966.

14. Vincent MB (2015) Vision and migraine. Headache 55: 595-599.

15. Van Dongen RM, Haan J (2019) Symptoms related to the visual system in migraine. Faculty Rev-12: 198.

16. Marzoli SB, Criscuoli A (2017) the role of visual system in migraine. Neurol Sci 38: 99-102.

17. Caminero-Rodriguez AB, Pareja JA (2008) Anatomical and neurochemical bases accounting for the frequent association between headaches and sleep: the hypnic headache paradigm. Rev Neurol 3047: 314320.

18. Shibata M, Suzuki N. (2013). Headache. Nihon Rinsho. 71: 2135-2140.
19. Kumar A, Arora R. (2019). Hemiplegic migraine. Stat Pearls Publishing 2019. Florida USA.

20. Deshmukh VD (2006) Retino-hypothalamicpineal hypothesis in the pathophysiology of primary headaches. Med Hypotheses. 66: 1146-1151.

21. Cortelli P, Pierangeli G (2007). Hypothalamus and headaches 28: S198-202.

22. Holland PR, Barloese M, Fahrenkrug J (2018) PACAP in hypothalamic regulation of sleep and circadian rhythm: importance for headache. J Headache Pain 19: 20.

23. Bourgeais-Rambur L, Beynac L, Villanueva L (2019) Brain network dysfunctions as substrates of primary headaches 213: 43-49.

24. Alstadhaug KB (2014) Histamine in migraine and brain. Headache 54: 246-259.

25. Worm J, Falkenberg K, Olesen J (2019) Histamine and migraine revisited: mechanisms and possible drug targets. J Headache Pain 20: 30.

26. Yuan H, Silberstein SD (2018) Histamine and migraine. Headache 58: 184-193.

27. Messlinger K, Fischer MJ, Lennerz JK (2011) Neuropeptide effects in the trigeminal system: pathophysiology and clinical relevance in migraine. Keio 60: 82-9.

28. Santos-Lasaosa S, Belvís R, Cuadrado ML, DíazInsa S, Gago-Veiga A, al et. (2019) Calcitonin generelated peptide in migraine: from pathophysiology to treatment. Neurologia. pii: S0213-4853-30075-1.

29. Sutherland HG, Albury CL, Griffiths LR (2019) Advances in genetics of migraine. J Headache Pain. 20: 72.

30. Freilinger T, Dichgans $M$ (2006) Genetics of migraine. Nervenarzt.77: 1186-1195.

Copyright: (c)2019 Orlando J.Castejón. This is an open-access article distributed under the terms of the Creative Commons Attribution License, which permits unrestricted use, distribution, and reproduction in any medium, provided the original author and source are credited. 